PHYSICAL REVIEW A 93, 039906(E) (2016)

\title{
Publisher's Note: Quantumness of spin-1 states [Phys. Rev. A 93, 012104 (2016)]
}

Fabian Bohnet-Waldraff, D. Braun, and O. Giraud

(Received 3 March 2016; published 16 March 2016)

DOI: 10.1103/PhysRevA.93.039906

This paper was published online on 8 January 2016 with a conversion error in Eq. (12).

Equation (12) should read as

$$
Q(|\psi\rangle) \geqslant-\sqrt{\frac{3}{8}} \lambda .
$$

The equation has been corrected as of 4 March 2016. The equation is incorrect in the printed version of the journal. 